\title{
On the $p$-Ranks of the Adjacency Matrices of Distance-Regular Graphs
}

RENÉ PEETERS

m.j.p.peeters@kub.nl

Department of Econometrics and Operations Research, Tilburg University, P.O. Box 90153, 5000 LE Tilburg, The Netherlands

Received March 22, 2000; Revised March 22, 2000

\begin{abstract}
Let $\Gamma$ be a distance-regular graph with adjacency matrix $A$. Let $I$ be the identity matrix and $J$ the all-1 matrix. Let $p$ be a prime. We study the $p$-rank of the matrices $A+b J-c I$ for integral $b, c$ and the $p$-rank of corresponding matrices of graphs cospectral with $\Gamma$.

Using the minimal polynomial of $A$ and the theory of Smith normal forms we first determine which $p$-ranks of $A$ follow directly from the spectrum and which, in general, do not. For the $p$-ranks that are not determined by the spectrum (the so-called relevant $p$-ranks) of $A$ the actual structure of the graph can play a rôle, which means that these $p$-ranks can be used to distinguish between cospectral graphs.

We study the relevant $p$-ranks for some classes of distance-regular graphs and try to characterize distance-regular graphs by their spectrum and some relevant $p$-rank.
\end{abstract}

Keywords: $\quad p$-rank, distance-regular graph, adjacency matrix, minimal polynomial

\section{Introduction}

In this paper we will deal with the following problem: Let $\Gamma$ be a regular graph with given spectrum, what can we say about the $p$-ranks of $A-c I$ (Notation: $r k_{p}(A-c I)$ ), or, more general, $A+b J-c I$, where $A$ is the adjacency matrix and $b$ and $c$ integers. Which of these $p$-ranks are completely determined by the spectrum and which are not? These $p$ ranks that are in general not determined by the spectrum of the graph can be used as an extra parameter to distinguish between cospectral graphs since for these $p$-ranks the actual structure of the graph can play a rôle. We will call these $p$-ranks the relevant $p$-ranks of the graph. If $\Gamma$ is cospectral with a distance-regular graph some of these remaining $p$-ranks can be determined if we furthermore assume that the graph is indeed distanceregular. This can only be the case for distance-regular graphs of diameter at least three since strong regularity follows from the spectrum. The $p$-ranks of the adjacency matrices of strongly regular graphs are studied by Brouwer and Van Eijl [3]. They examine which $p$-ranks follow directly from the spectrum (parameters) of the strongly regular graph and calculate the remaining (relevant) $p$-ranks for many classes of strongly regular graphs such as triangular graphs, lattice graphs, Paley graphs and the sporadic graphs. In [11] the present author investigated the characterization of strongly regular graphs by their spectrum and some relevant $p$-rank of the graph. Especially so-called "rigidity theorems" for strongly 
Table 1. The relevant $p$-ranks of all graphs cospectral with the Hamming graph $H(3,3)$.

\begin{tabular}{rrrrrr}
\hline & $|\operatorname{Aut}(\Gamma)|$ & $r k_{3}(A)$ & $r k_{2}(A+I)$ & \\
\hline 1 & 288 & $13-$ & 19 & 1 & \\
2 & 72 & $14-$ & 17 & 1 & \\
3 & 1296 & $12-$ & 19 & 1 & $H^{\prime}(3,3)$ \\
4 & 1296 & $14-$ & 13 & 1 & $H(3,3)$ \\
\hline
\end{tabular}

regular graphs, which are characterizations of strongly regular graphs by their parameters and the minimality of some relevant $p$-rank, got attention.

In this paper we will concentrate on distance-regular graphs of diameter at least three and graphs cospectral with these and we want to derive similar results for these graphs as are obtained for strongly regular graphs. In the next section we will examine which $p$-ranks of some regular graph follow from its spectrum, generalizing results from [3]. Our main tool to determine these $p$-ranks is the minimal polynomial of the adjacency matrix of the graph considered as a matrix over $I F_{p}$. We will first mention some properties of the minimal polynomial and then show how it determines most of the $p$-ranks of some regular graph, given its spectrum. If not all eigenvalues are integral some extra $p$-ranks can be determined from the spectrum using the theory of Smith normal forms. In the third section we consider the Hamming and Doob graphs as our main example. Some more examples are considered in the last section. We try to determine the relevant $p$-ranks for these graphs and try to characterize some of these graphs by their spectrum and the minimality of some relevant $p$-rank. We use the results of Haemers and Spence [8], who determined all graphs cospectral with a distance-regular graph with at most 30 vertices, as illustrative examples. This paper is based on the author's Ph.D. thesis [10].

Example 1.1 The Hamming graph $H(3,3)$ on 27 vertices has spectrum $6^{1}, 3^{6}, 0^{12},-3^{8}$. Given a graph with this spectrum, $\Gamma$ say, with adjacency matrix $A$, all $p$-ranks of $A-c I$ ( $c$ integral) are determined by the spectrum, except possibly for $r k_{3}(A)$ and $r k_{2}(A+I)$. According to Haemers and Spence [8] there are four graphs with this spectrum. One of these is the graph $H^{\prime}(3,3)$ which is the graph defined on the 27 lines (3-cliques) of the Hamming graph $H(3,3)$, where two lines are defined to be adjacent if they intersect. The relevant $p$-ranks of the four graphs cospectral with $H(3,3)$ are shown in Table 1.

Notice that $H(3,3)$ and $H^{\prime}(3,3)$ have the largest automorphism group and can both be characterized by their spectrum and the minimality of some relevant $p$-rank. $H(3,3)$ has the smallest 2-rank (of $A+I$ ) and $H^{\prime}(3,3)$ has the smallest 3-rank.

\section{Preliminaries}

\subsection{The minimal polynomial}

Let $F$ be any field and $A$ an $n \times n$-matrix over $F$. A polynomial $f(x) \in F[x]$ is called an annihilating polynomial of $A$ if $f(A)=O$. The minimal polynomial of $A$ is the unique monic annihilating polynomial of $A$ that has minimal degree. The characteristic polynomial 
of $A$ is the polynomial $\operatorname{det}(x I-A)$. Let $\varphi_{0}(x)$ be the minimal polynomial of $A$ and let $c \in F$, then equivalent are:

1. $r k(A-c I)<n$,

2. $c$ is a root of $\operatorname{det}(x I-A)$,

3. $c$ is a root of $\varphi_{0}(x)$.

Let $\varphi_{0}(x)=\left(x-\lambda_{1}\right)^{h_{1}} \ldots\left(x-\lambda_{n}\right)^{h_{n}} \varphi_{1}(x)$, where $\varphi_{1}(x)$ has no roots in $F$ and let $\operatorname{det}(x I-A)=\left(x-\lambda_{1}\right)^{m_{1}} \ldots\left(x-\lambda_{n}\right)^{m_{n}} f(x)$ where $f(x)$ has no roots in $F$, then $\varphi_{1}(x)$ divides $f(x)$ and there exists a regular $v \times v$-matrix $S$ such that

$$
S^{-1} A S=\operatorname{diag}\left(A_{1}, \ldots, A_{n}, B\right)
$$

where $A_{i}$ is an $m_{i} \times m_{i}$-matrix such that $\left(A_{i}-\lambda_{i} I\right)$ is nilpotent with index $h_{i}$ and $B$ is an $\left(n-\sum m_{i}\right) \times\left(n-\sum m_{i}\right)$-matrix that has no eigenvalues in $F$. Now $r k\left(\left(A-\lambda_{i} I\right)^{h_{i}}\right)=$ $n-m_{i}$ and

$$
n-m_{i}+h_{i}-1 \leq r k\left(A-\lambda_{i} I\right) \leq n-\frac{m_{i}}{h_{i}}
$$

so $\lambda_{i}$ is a simple root of $\varphi_{0}(x)$ if and only if $r k\left(A-\lambda_{i} I\right)=n-m_{i}$.

Furthermore, $\operatorname{ker}\left(\left(A-\lambda_{i} I\right)^{h_{i}}\right)+\left\langle\left(A-\lambda_{i} I\right)^{h_{i}}\right\rangle=F^{n}$ and $\operatorname{ker}\left(\left(A-\lambda_{i} I\right)^{h_{i}}\right) \cap\left\langle\left(A-\lambda_{i} I\right)^{h_{i}}\right\rangle$ $=\{\mathbf{0}\}$.

For a matrix $M \in F^{m \times n}$ we write $\langle M\rangle$ for the row space of $M$ over $F$ and $\operatorname{ker}(M)$ for the kernel of $M$ over $F$. If $F=I F_{p}$ we use the notation $\langle M\rangle_{p}$ and $\operatorname{ker}_{p}(M)$; also if $M$ is integral.

In this paper we will only consider the minimal polynomial of the adjacency matrix of a graph considered as a matrix over $I R$ or $I F_{p}$. Dealing with this situation, we have the following lemma:

Lemma 2.1 (cf. [5]) Let $A$ be an integral $n \times n$-matrix, then the minimal polynomial of A over IR, $\varphi_{0}(x)$ say, has integral coefficients.

So if we consider $A$ as a matrix over $I F_{p}, \varphi_{0}(x)(\bmod p)$ is an annihilating polynomial of $A$ and the minimal polynomial of $A$ modulo $p$ divides $\varphi_{0}(x)(\bmod p)$.

\subsection{Distance-regular graphs}

A connected graph $\Gamma$ is called distance-regular if it is regular of valency $k$, and if for any two points $\gamma, \delta \in \Gamma$ at (graph) distance $i$, there are precisely $c_{i}$ neighbors of $\delta$ at distance $i-1$ from $\gamma$ and $b_{i}$ neighbors of $\delta$ at distance $i+1$ from $\gamma$. The sequence

$$
\iota(\Gamma)=\left\{b_{0}, b_{1}, \ldots, b_{d-1} ; c_{1}, c_{2}, \ldots, c_{d}\right\}
$$

where $d$ is the diameter of $\Gamma$, is called the intersection array of $\Gamma$; the numbers $c_{i}, b_{i}$ and $a_{i}$, where

$$
a_{i}=k-b_{i}-c_{i}
$$


is the number of neighbors of $\delta$ at distance $i$ from $\gamma$, are called the intersection numbers of $\Gamma$. By definition we have that

$$
b_{0}=k, \quad b_{d}=c_{0}=0, \quad c_{1}=1 .
$$

For a graph $\Gamma$ we can define the $i$-th distance matrix $A_{i}(\Gamma)$ (or just $A_{i}$ ) of $\Gamma$ as the $v \times v$ zero-one matrix with $\left(A_{i}\right)_{x y}=1$ if and only if $x$ and $y$ are at distance $i$ in $\Gamma$. The adjacency matrix $A$ of $\Gamma$ is the matrix $A_{1}$. The eigenvalues of a graph are the eigenvalues of its adjacency matrix. One easily checks that the matrices $A_{i}$ satisfy the relations

$$
\begin{aligned}
A_{0}= & I, \quad A_{1}=A, \\
A A_{i}= & c_{i+1} A_{i+1}+a_{i} A_{i}+b_{i-1} A_{i-1} \quad(i=1,2, \ldots, d), \\
& A_{0}+A_{1}+\cdots+A_{d}=J .
\end{aligned}
$$

It follows that the matrices $A_{i}$ can be written as polynomials in $A$ of degree $i$,

$$
A_{i}=v_{i}(A)
$$

where the polynomials $v_{i}$ are defined recursively by

$$
\begin{aligned}
v_{0}(x) & =1, \quad v_{1}(x)=x, \\
c_{i+1} v_{i+1}(x) & =\left(x-a_{i}\right) v_{i}(x)-b_{i-1} v_{i-1}(x) \quad(i=1,2, \ldots, d-1) .
\end{aligned}
$$

Since the $d+1$ matrices $A_{i}$ are linearly independent the minimal polynomial of $A$ (over $I R$ ) has degree at least $d+1$, so $A$ has at least $d+1$ eigenvalues. By (1) and (2) we find that

$$
A_{i} A_{j}=\sum_{t=0}^{d} p_{i j}^{t} A_{t}
$$

for certain numbers $p_{i j}^{t}$. By comparison of the $x y$-entries we see that for vertices $x$ and $y$ at distance $t$, the number $p_{i j}^{t}$ equals the number of vertices $z$ with $d(x, z)=i$ and $d(y, z)=j$ for $i, j, t=0,1, \ldots, d$. In particular the $p_{i j}^{t}$ are non-negative integers. It follows that the $A_{i}$ generate a $d+1$-dimensional commutative algebra $\mathcal{A}$ of symmetric matrices, called the Bose-Mesner algebra of the distance-regular graph. We denote with $\mathcal{A}_{p}$ the modular Bose-Mesner algebra, that is the algebra generated by the $A_{i}$ over $I F_{p}$.

Since the matrices $A_{i}$ commute, they can be diagonalized simultaneously over the complex numbers. Therefore $\mathcal{A}$ is semi-simple and has a unique basis of minimal idempotents $E_{0}=\frac{1}{v} J, E_{1}, E_{2}, \ldots, E_{d}$. Now the matrix of eigenvalues $P$ is defined by:

$$
A_{j}=\sum_{i=0}^{d} P_{i j} E_{i}
$$

Let $v_{i}$ be the valency of $A_{i}$ (so $v_{i}=P_{0 i}$ ) and let $m_{i}$ be the multiplicity of the $i$-th eigenvalue (so $m_{i}=r k\left(E_{i}\right)$ ). 
The intersection matrix of $\Gamma$ is the tridiagonal matrix

$$
L_{1}=\left(\begin{array}{ccccccc}
a_{0} & b_{0} & & & & & \\
c_{1} & a_{1} & b_{1} & & & & \\
& c_{2} & a_{2} & b_{2} & & & \\
& & c_{3} & \cdot & \cdot & & \\
& & & \cdot & \cdot & \cdot & \\
& & & & \cdot & \cdot & b_{d-1} \\
& & & & & c_{d} & a_{d}
\end{array}\right) .
$$

Similarly the intersection matrices $L_{0}, L_{1}, \ldots, L_{d}$ can be defined by

$$
\left(L_{i}\right)_{k j}=p_{i j}^{k}
$$

Multiplication by $A$ defines a linear map on $\mathcal{A}\left(\mathcal{A}_{p}\right)$. With respect to the basis $\left\{A_{0}, A_{1}, \ldots\right.$, $\left.A_{d}\right\}$ of $\mathcal{A}$ this linear map has matrix $L_{1}$. More generally, let $f(x)$ be a polynomial, then multiplication by $f(A)$ defines a linear map on $\mathcal{A}$ with matrix $f\left(L_{1}\right)$. It follows that $f(A)=O$ if and only if $f\left(L_{1}\right)=O$ and in fact $f(A)=O$ if and only if the first column of $f\left(L_{1}\right)$ is all-zero, so $A$ and $L_{1}$ have the same minimal polynomial, over $I R$ as well as over $I F_{p}$ and in fact the same is true for $A_{i}$ and $L_{i}$ in general. Since the minimal polynomial of $A$ over $I R$ has rank at least $d+1$ which is the order of $L_{1}$, the minimal polynomial over $I R$ of $A$ and $L_{1}$ must be $\operatorname{det}\left(x I-L_{1}\right)$. So $A$ and $L_{1}$ have the same $d+1$ eigenvalues which can be calculated from the intersection array only. Also the multiplicities of these eigenvalues can be calculated from the intersection array only (cf. [2] Theorem 4.1.4).

Let $P_{1}$ be the matrix got from the matrix of eigenvalues $P$ by deleting its first row and column. Since the sum of the columns of $P$ is equal to the vector $(v, 0, \ldots, 0)^{T}$ we have $\operatorname{det} P=v \operatorname{det} P_{1}$. Now

$$
\left|\operatorname{det} P_{1}\right|^{2}=v^{d-1} \prod_{i=0}^{d} \frac{v_{i}}{m_{i}} .
$$

It can be proven that the right side here is an integer. It is known as the Frame quotient (cf. [2]).

For determining the minimal polynomial of the adjacency matrix of a distance-regular graph over $I F_{p}$, we have the following lemma.

Lemma 2.2 Let $A$ be the adjacency matrix of a distance-regular graph with intersection matrix $L_{1}$ and let $p$ be a prime, then, when calculating modulo $p, A$ and $L_{1}$ have the same minimal polynomial. Let $e:=\min \left\{d+1, i \mid c_{i} \equiv 0(\bmod p), i>0\right\}$ and L the $e \times e$ submatrix of $L_{1}$ consisting of the first e rows and columns, then this minimal polynomial is equal to $\operatorname{det}(x I-L)$. 
Proof: Let $f(x)$ be a polynomial with integral coefficients, then $f(A) \equiv O(\bmod p)$ if and only if $f\left(L_{1}\right) \equiv O(\bmod p)$. In fact $f(A) \equiv O(\bmod p)$ if and only if the first column of $f\left(L_{1}\right)(\bmod p)$ is all-zero. Let

$$
L_{1} \quad(\bmod p)=\left(\begin{array}{cc}
L & M \\
O & L^{\prime}
\end{array}\right), \quad \text { then } f\left(L_{1}\right) \quad(\bmod p)=\left(\begin{array}{cc}
f(L) & M^{\prime} \\
O & f\left(L^{\prime}\right)
\end{array}\right)
$$

for each polynomial $f(x)$. Since the matrices $L^{0}=I, L^{1}, L^{2}, \ldots, L^{e-1}$ are clearly linearly independent, the minimal polynomial of $L$ over $I F_{p}$ has degree at least $e$, so it must be equal to $\operatorname{det}(x I-L)(\bmod p)$.

For determining $p$-ranks of integral matrices from Bose-Mesner algebras the following theorem by Godsil [6] is useful and is in fact a much more general tool than the lemma above.

Theorem 2.3 (Godsil) Let $F$ be a finite field of characteristic $p$ that contains the eigenvalues of all adjacency matrices of an association scheme. Then the Bose-Mesner algebra of this association scheme over $F$ is semisimple if and only if $p$ does not devide $|\operatorname{det} P|^{2}=$ $v^{d+1} \prod_{i=0}^{d} \frac{v_{i}}{m_{i}}$.

Corollary 2.4 Let $\mathcal{A}$ be the Bose-Mesner algebra of an association scheme and let $p$ be a prime not dividing $|\operatorname{det} P|^{2}$. Then the minimal polynomial of any matrix in $\mathcal{A}_{p}$ does not contain multiple roots.

So for any integral matrix $M$ from the Bose-Mesner algebra of an association scheme the only $p$-ranks of $M$ that are not necessarily determined by the characteristic polynomial of $M$ are those for which $p$ divides $|\operatorname{det} P|^{2}$.

\section{3. $\quad$-Ranks of integral matrices with given spectrum}

In this section we consider the following problem: Given the spectrum of a symmetric integral matrix, $M$ say, of order $v$ and with constant row sum (think of $M=A+b J$ for some integer $b$ and $A$ the adjacency matrix of some regular graph with prescribed spectrum). Which $p$-ranks of $M-c I$ (for integral $c$ ) are determined by the spectrum of $M$ and which are not (in general)?

First of all, if $\mathbf{1} \notin\langle M\rangle_{p}$ and $\mathbf{1} \in\langle M+b J\rangle_{p}$ for some $b \neq 0$, then $\mathbf{1} \in\langle M+b J\rangle_{p}$ for all $b \neq 0(\bmod p)$. Thus, either $r k_{p}(M+b J)$ is independent of $b$, or there is precisely one value of $b$ for which this rank is one lower than for all other values. If $p$ does not divide $v$, we are in the latter case.

Since the spectrum of $M$ is known, also its minimal polynomial over $I R, \varphi_{0}(x)$ say, is known as well as its characteristic polynomial. Most $p$-ranks of the matrices $M-c I$ follow from the properties of the minimal polynomial of $M$ over $I F_{p}$. More precisely, let $\theta_{0}, \theta_{1}, \ldots, \theta_{n-1}$ be the $n$ distinct eigenvalues of $M$ with $\theta_{0}$ equal to the row sum of $M$ having multiplicity equal to 1 . If $c$ is not a multiple root of $\prod_{i=1}^{n-1}\left(x-\theta_{i}\right)(\bmod p)$ then the $p$-rank 
of $M-c I$ is determined by the spectrum of $M$ as is shown in the next lemma. Since the proof is technical and straightforward (see [10]) it is omitted.

Lemma 2.5 Let $M$ be a symmetric integral matrix of order $v$ with constant row sum $\theta_{0}$ and $n$ distinct eigenvalues $\theta_{0}, \theta_{1}, \ldots, \theta_{n-1}$ with $m_{0}=1$. Let $f(x)=\prod_{i=1}^{n-1}\left(x-\theta_{i}\right)$ and $\varphi_{0}(x)=\left(x-\theta_{0}\right) f(x)$, which are both polynomials with integral coefficients. Let $c$ be an integer and $p$ a prime number.

If $c$ is not a root of $f(x)(\bmod p)$, then

$$
\begin{aligned}
r k_{p}(M-c I)= & v \quad \text { if } p \text { does not divide } \theta_{0}-c, \\
v-1 & \text { if } p \text { divides } \theta_{0}-c .
\end{aligned}
$$

If $c$ is a simple root of $f(x)(\bmod p)$, and the characteristic polynomial of $M$ modulo $p$ contains $m_{c}$ factors $(x-c)$ then

$$
\begin{array}{cl}
r k_{p}(M-c I)= & v-m_{c} \quad \text { if } p \text { does not divide } \theta_{0}-c, \\
v-m_{c} & \text { if } p \text { divides both } \theta_{0}-c \text { and } \frac{1}{v} f\left(\theta_{0}\right), \\
v-m_{c}+1 & \text { if } p \text { divides } \theta_{0}-c \text { but not } \frac{1}{v} f\left(\theta_{0}\right) .
\end{array}
$$

Corollary 2.6 Let $M$ be a symmetric, integral $v \times v$-matrix with constant row sum $\theta_{0}$ and $n$ distinct integral eigenvalues $\theta_{0}^{m_{0}=1}, \theta_{1}^{m_{1}}, \theta_{2}^{m_{2}}, \ldots, \theta_{n-1}^{m_{n-1}}$, where the exponents denote the multiplicities and $\sum_{i=0}^{n-1} m_{i}=, v$. The only p-ranks of matrices $M-c I$ (for integral $\left.c\right)$ that are not necessarily determined by the spectrum are:

$$
r k_{p}\left(M-\theta_{i} I\right) \quad \text { for } p \mid\left(\theta_{j}-\theta_{i}\right) \quad \text { with } i \neq j \in\{1,2, \ldots, n-1\}
$$

Corollary 2.7 Let $M$ be a symmetric integral $v \times v$-matrix with constant row sum $\theta_{0}$ and $n$ distinct eigenvalues $\theta_{0}^{m_{0}=1}, \theta_{1}^{m_{1}}, \ldots, \theta_{n-3}^{m_{n-3}}, \theta_{n-2}=\frac{1}{2}(a+\sqrt{b})^{m_{n-2}}, \theta_{n-1}=\frac{1}{2}(a-\sqrt{b})^{m_{n-2}}$ with integral $\theta_{1}, \ldots, \theta_{n-3}, a, b, \frac{1}{4}\left(a^{2}-b\right)$. Let $p$ be a prime and $f(x)=\prod_{i=1}^{n-1}\left(x-\theta_{i}\right)=$ $\prod_{i=1}^{n-3}\left(x-\theta_{i}\right)\left(x^{2}-a x+\frac{1}{4}\left(a^{2}-b\right)\right)$. Then all p-ranks of $M-c I($ for integral $c)$ are determined by the spectrum of $M$, except possibly if modulo $p f(x)$ contains at least two factors $(x-c)$. So the $p$-ranks that do not follow from the minimal polynomial of $M$ are:

$$
\begin{array}{ll}
r k_{p}\left(M-\theta_{i} I\right) & \text { with } p \mid\left(\theta_{i}-\theta_{j}\right) \\
r k_{p}\left(M-\theta_{i} I\right) & \text { with } p \mid\left(\theta_{i}-\theta_{n-2}\right)\left(\theta_{i}-\theta_{n-1}\right) \\
r k_{2}\left(M-\theta_{n-2} \theta_{n-1} I\right) & \text { for } i \in\{1,2, \ldots, n-3\} \\
r k_{p}\left(M-\frac{1}{2}\left(\theta_{n-2}+\theta_{n-1}\right) I\right) & \text { if } \theta_{n-2}+\theta_{n-1} \text { is even }
\end{array}
$$


Sometimes we can reduce this set of undecided $p$-ranks using the theory of Smith normal forms. More precisely we will use the following lemma (cf. [3]):

Lemma 2.8 Let $M$ be a non-singular integral matrix of order $n$ and suppose $p^{k} \| \operatorname{det} M$ (that means $p^{k}$ divides $\operatorname{det} M$ but $p^{k+1}$ does not). Then $r k_{p}(M) \geq n-k$.

Lemma 2.9 Let $M$ be a matrix as in Corollary 2.7. Let $p$ be a prime that divides $\left(\theta_{n-2}-\right.$ $\left.\theta_{n-1}\right)^{2}$ precisely once and let $A:=M-\frac{1}{2}\left(\theta_{n-2}+\theta_{n-1}\right)$ I. If none of $\theta_{1}, \theta_{2}, \ldots, \theta_{n-3}$ is a solution of $x^{2}-a x+\frac{1}{4}\left(a^{2}-b\right)$ then

if $\theta_{0} \not \equiv \frac{1}{2}\left(\theta_{n-2}+\theta_{n-1}\right)(\bmod p)$ then $r k_{p}(A)=v-m_{n-2}$;

if $\theta_{0} \equiv \frac{1}{2}\left(\theta_{n-2}+\theta_{n-1}\right)(\bmod p)$ and $p$ does not divide $v$ then $r k_{p}(A)=v-m_{n-2}-1$;

if $\theta_{0} \equiv \frac{1}{2}\left(\theta_{n-2}+\theta_{n-1}\right)(\bmod p)$ and $p$ does divide $v$ then $r k_{p}(A)=v-m_{n-2}-1$ if

$\frac{1}{v} \prod_{i=1}^{n-1}\left(\theta_{0}-\theta_{i}\right) \equiv 0(\bmod p)$ and $r k_{p}(A)=v-m_{n-2}$ otherwise.

Proof: If $\theta_{0} \not \equiv \frac{1}{2}\left(\theta_{n-2}+\theta_{n-1}\right) \quad(\bmod p)$ then $p^{m_{n-2}} \| \operatorname{det}(A)\left(\operatorname{so} r k_{p}(A) \geq v-m_{n-2}\right)$ and $r k_{p}\left((A)^{2}\right)=v-2 m_{n-2}\left(\right.$ so $\left.r k_{p}(A) \leq v-m_{n-2}\right)$ and hence $r k_{p}(A)=v-m_{n-2}$.

If $\theta_{0} \equiv \frac{1}{2}\left(\theta_{n-2}+\theta_{n-1}\right)$ then $r_{p}(A)$ is equal to either $v-m_{n-2}$ or $v-m_{n-2}-1$. If furthermore $v \not \equiv 0 \quad(\bmod p)$ then $r k_{p}(A)=v-m_{n-2}-1$ (Indeed $\mathbf{1} \notin\langle A\rangle_{p}$ and $\mathbf{1} \in\langle A+$ $J\rangle_{p}$.).

Now suppose that $v \equiv 0 \quad(\bmod p)$ and $\theta_{0} \equiv \frac{1}{2}\left(\theta_{n-2}+\theta_{n-1}\right)$, then we still have to prove that

$$
\begin{array}{cl}
r k_{p}(A)=v-m_{n-2}-1 & \text { if } \frac{1}{v} \prod_{i=1}^{n-1}\left(\theta_{0}-\theta_{i}\right) \equiv 0 \quad(\bmod p) \\
v-m_{n-2} & \text { otherwise. }
\end{array}
$$

Indeed, $\prod_{i=1}^{n-3}\left(M-\theta_{i} I\right)$ has $p$-rank $1+2 m_{n-2}$. If $A$ has $p$-rank $v-m_{n-2}$ then $r k_{p}\left(A^{2}\right)=$ $v-2 m_{n-2}$, so $\prod_{i=1}^{n-1}\left(M-\theta_{i} I\right)=\frac{1}{v} \prod_{i=1}^{n-1}\left(\theta_{0}-\theta_{i}\right) J \not \equiv O(\bmod p)$. If $\frac{1}{v} \prod_{i=1}^{n-1}\left(\theta_{0}-\theta_{i}\right) \not \equiv 0$ $(\bmod p)$ then $\mathbf{1} \in\left\langle A^{2}\right\rangle_{p}$ and if $\left(\theta_{0}-\theta_{n-2}\right)\left(\theta_{0}-\theta_{n-1}\right)$ contains precisely $e$ factors $p$, also $v$ contains precisely $e$ factors $p$. Let $\underline{x}$ and $y$ be two vectors such that $A^{2} y^{T}=\mathbf{1}^{T}$ and $\underline{x} A=\mathbf{1}$. Then $\underline{x} \mathbf{1}^{T}=\underline{x} A^{2} y^{T}=0$, so there exists a vector $\underline{x}$ such that $\underline{x} A=\mathbf{1}$ and $\underline{x} \mathbf{1}^{T}=0$. It follows that $A$ has the same $p$-rank as the $(v+1) \times(v+1)$-matrix $B$ defined by

$$
B:=\left(\begin{array}{c|c}
\theta_{0}-\frac{1}{2}\left(\theta_{n-2}+\theta_{n-1}\right) & \mathbf{1} \\
\hline \mathbf{1}^{T} & A
\end{array}\right)
$$

We have that $p$ divides $v$ precisely once since $\left(\theta_{0}-\theta_{n-2}\right)\left(\theta_{0}-\theta_{n-1}\right)=\left(\theta_{0}-\frac{1}{2}\left(\theta_{n-2}+\right.\right.$ $\left.\left.\theta_{n-1}\right)\right)^{2}-\frac{\left(\theta_{n-2}-\theta_{n-1}\right)^{2}}{4}$. If $\eta_{i}=\theta_{i}-\frac{1}{2}\left(\theta_{n-2}+\theta_{n-1}\right)$ for $i=0,1, \ldots, n-1$ are the eigenvalues of $A$, then $B$ has spectrum:

$$
\left(\eta_{0}+\sqrt{v}\right)^{1},\left(\eta_{0}-\sqrt{v}\right)^{1}, \eta_{1}^{m_{1}}, \ldots, \eta_{n-1}^{m_{n-1}}
$$


It follows that $\operatorname{det}(B)$ contains $m_{n-2}+1$ factors $p\left(1\right.$ factor from $\left(\eta_{0}+\sqrt{v}\right)\left(\eta_{0}-\sqrt{v}\right)=$ $\eta_{0}^{2}-v$ and $m_{n-2}$ factors from $\left(\eta_{n-2} \eta_{n-1}\right)^{m_{n-2}}$ since $\left.\eta_{n-2} \eta_{n-1}=-\frac{\left(\theta_{n-2}-\theta_{n-1}\right)^{2}}{4}\right)$ so $r k_{p}(A)=$ $r k_{p}(B) \geq v-m_{n-2}$. It follows that $r k_{p}(A)=v-m_{n-2}$.

So if $M$ is a matrix as in Corollary 2.7 we are left with the following $p$-ranks:

$$
\begin{array}{ll}
r k_{p}\left(M-\theta_{i} I\right) & \text { with } p \mid\left(\theta_{i}-\theta_{j}\right) \\
r k_{p}\left(M-\theta_{i} I\right) & \text { for } i \neq j \in\{1,2, \ldots, n \\
r k_{2}\left(M-\theta_{n-2} \theta_{n-1} I\right) & \text { with } p \mid\left(\theta_{i}-\theta_{n-2}\right)\left(\theta_{i}-\theta_{n-1}\right) \\
r k_{p}\left(M-\frac{1}{2}\left(\theta_{n-2}+\theta_{n-1}\right) I\right) & \text { for } i \in\{1,2, \ldots, n-3\} \\
& \text { for odd } \theta_{n-2}+\theta_{n-1} \text { is even }
\end{array}
$$

Example 2.1 According to Haemers and Spence [8] there are ten graphs on 24 vertices with spectrum $7^{1}, \sqrt{7}^{8},-1^{7},-\sqrt{7}^{8}$. One of these, the so-called Klein graph, is distanceregular with intersection array $\{7,4,1 ; 1,2,7\}$. The $p$-ranks that are still open in this case are $r k_{2}(A+I)$ and $r k_{3}(A+I)$. Table 2 denotes the ranks we find for the ten graphs with the mentioned spectrum (The number after the value for the rank is the unique $b_{0}$ such that $r k_{p}\left(A-c I+b_{0} J\right)=r k_{p}(A-c I+b J)-1$ for all $b \neq b_{0}$, or '-' in case $r k_{p}(A-c I+b J)$ is independent of $b$ ).

Notice that 7 divides $\left(\theta_{1}-\theta_{3}\right)^{2}=28$ but $7^{2}=49$ does not divide 28 , so for all 10 graphs cospectral with the Klein graph $r k_{7}(A)$ can be determined using the last lemma. We have $\theta_{0} \equiv \frac{1}{2}(\sqrt{7}+(-\sqrt{7}))(\bmod 7)$ and 7 does not divide $v=24$, so $r k_{7}(A)=$ $24-8-1=15$.

Since $\mid$ det $\left.P\right|^{2}=2^{8} * 3^{4} * 7$ Godsil's theorem does not reduce the set of relevant $p$-ranks for the Klein graph.

Table 2. The relevant $p$-ranks of all graphs cospectral with the Klein graph.

\begin{tabular}{rrrcrl}
\hline & $\mid$ Aut $(\Gamma) \mid$ & $r k_{2}(A+I)$ & $r k_{3}(A+I)$ & \\
\hline 1 & 2 & 14 & $16-$ & \\
2 & 12 & $14-$ & $16-$ & \\
3 & 3 & 15 & 1 & $16-$ & \\
4 & 2 & 13 & 1 & $16-$ & \\
5 & 16 & 12 & - & $16-$ & \\
6 & 8 & 11 & 1 & $16-$ & \\
7 & 6 & 13 & 1 & $16-$ & \\
8 & 6 & 15 & 1 & $16-$ & \\
9 & 42 & 15 & 1 & $16-$ & \\
10 & 336 & 9 & 1 & $16-$ & Klein graph \\
\hline
\end{tabular}




\section{Hamming and Doob graphs}

\subsection{Definitions}

The Kronecker product of two matrices $A$ and $B$ is the matrix with blocks $a_{i j} B$ and is denoted by $A \otimes B$. By an easy exercise $(A \otimes P)(B \otimes Q)=A B \otimes P Q$ where $A$ and $B$ respectively $P$ and $Q$ have fitting sizes.

Let $G_{1}=\left(V_{1}, E_{1}\right)$ and $G_{2}=\left(V_{2}, E_{2}\right)$ be two graphs with adjacency matrices $A_{1}$ and $A_{2}$ respectively. The direct product of $G_{1}$ and $G_{2}$ is the graph with vertex set $V_{1} \times V_{2}$ and two vertices $\left(v_{1}, w_{1}\right)$ and $\left(v_{2}, w_{2}\right)$ are adjacent if and only if $\left(\left(v_{1}=v_{2}\right) \wedge\left(w_{1}, w_{2}\right) \in E_{2}\right)$ or $\left(\left(w_{1}=w_{2}\right) \wedge\left(v_{1}, v_{2}\right) \in E_{1}\right)$. So by definition the direct product of $G_{1}$ and $G_{2}$ has adjacency matrix $I \otimes A_{1}+A_{2} \otimes I$. Usually this is called the Kronecker sum of $A_{1}$ and $A_{2}$ (notation: $\left.A_{1} \oplus A_{2}\right)$. If $\left\{\lambda_{i}\right\}$ and $\left\{\mu_{j}\right\}$ are the eigenvalues of $A_{1}$ and $A_{2}$ respectively, then $\left\{\lambda_{i}+\mu_{j}\right\}$ are the eigenvalues of $A_{1} \oplus A_{2}$.

Let $X$ be a finite set of cardinality $q \geq 2$. The Hamming graph $\Gamma$ (with diameter $d$ on $X$ ) has vertex set $X^{d}$, the cartesian product of $d$ copies of $X$; two vertices of $\Gamma$ are adjacent whenever they differ in precisely one coordinate. An equivalent definition is that a Hamming graph is the direct product of $d$ copies of a complete graph on $q$ vertices. Because only $d$ and $q$ will be relevant here, we denote $\Gamma$ as well as its adjacency matrix by $H(d, q)$.

Hamming graphs are distance-regular with diameter $d$ and parameters $b_{j}=(d-j)$ $(q-1), c_{j}=j$ and $a_{j}=j(q-2)$ for $0 \leq j \leq d$. As distance-regular graphs they are uniquely determined by their parameters, except when $q=4$. In this case the Doob graphs, which we will denote here by $D(n, m)$ and which are defined as the direct product of a Hamming graph $H(n, 4)$ with $m$ copies of a Shrikhande graph, are distance-regular with the same parameters as $H(n+2 m, 4)$. There are no other exceptions, see [2] Section 9.2. Hamming graphs have eigenvalues $\theta_{j}=q(d-j)-d$ with multiplicities $f_{j}=\left(\begin{array}{c}d \\ j\end{array}\right)(q-1)^{j}(j=0,1, \ldots, d)$. Because the eigenvalues of a distance-regular graph are determined by its parameters the Doob graph $D(n, m)$ has the same eigenvalues as $H(n+2 m, 4)$.

\section{2. $\quad$ The p-ranks}

In this section we will determine the $p$-ranks of matrices $A-c I$ for integral $c$ where $A$ is the adjacency matrix of a Pseudo Hamming graph, that is a distance-regular graph with the same parameters as some Hamming graph $H(d, q)$. It turns out that almost all of these $p$-ranks follow from the minimal polynomial of $A$, considered as matrix over $I F_{p}$. In order to determine the remaining $p$-ranks we have to use the structure of the considered Hamming or Doob graph.

Theorem 3.1 Let $A$ be the adjacency matrix of a distance-regular graph with the same parameters as the Hamming graph $H(d, q)$, then

$$
\begin{array}{ll}
A^{p}-A \equiv O(\bmod p) & \text { if } p \nmid q \\
A^{p}+d I \equiv O(\bmod p) & \text { if } p \mid q
\end{array}
$$


Proof: Let $L_{1}$ be the intersection matrix of the distance-regular graph then by Lemma 2.2 $A$ and $L_{1}$ have the same minimal polynomial, so the theorem is trivial if $d<p$ since then the characteristic polynomial of $L_{1}$ divides $x^{p}-x$ if $p \nmid q$ and $x^{p}+d$ if $p \mid q$. If $d \geq p$ then $c_{p} \equiv 0(\bmod p)$, so $A$ has minimal polynomial $\varphi_{0}(x)$ of degree $p$. If $p \mid q$ then all eigenvalues are equal to $-d$ modulo $p$, so $\varphi_{0}(x)=(x+d)^{p}=x^{p}+d$. If $p \nmid q$ then modulo $p$ the first $p$ eigenvalues of $L_{1}$ are all different, so $\varphi_{0}(x)$ contains a factor $x-c$ for each $c \in I F_{p}$ which implies that $\varphi_{0}(x)=x^{p}-x$.

More precisely, the minimal polynomial $\varphi_{0}(x)$ of $A$ over $I F_{p}$ is

$$
\varphi_{0}(x)= \begin{cases}\prod_{i=0}^{d}\left(x-\theta_{i}\right) & \text { if } p \nmid q \text { and } d<p \\ x^{p}-x & \text { if } p \nmid q \text { and } d \geq p \\ (x+d)^{d} & \text { if } p \mid q \text { and } d<p \\ x^{p}+d & \text { if } p \mid q \text { and } d \geq p\end{cases}
$$

Since each element of $I F_{p}$ is a simple root of $x^{p}-x$, almost all $p$-ranks of Pseudo Hamming graphs follow from the eigenvalues and the intersection numbers. In particular we have:

Corollary 3.2 Let $A$ be the adjacency matrix of a distance regular graph with the same parameters as a Hamming graph $H(d, q)$. Let $c$ be an integer and $p$ a prime, then

$$
\begin{aligned}
r k_{p}(A-c I) & =q^{d}-\sum_{\theta_{i}-c \equiv 0(\bmod p)} f_{i} & & \text { if } p \nmid q \\
r k_{p}(A-c I) & =q^{d} & & \text { if } p \mid q \text { and } c+d \not \equiv 0(\bmod p) \\
r k_{p}\left((A+d I)^{e}\right) & \leq \frac{p-e}{p} q^{d} & & \text { for } 0 \leq e \leq p \text { if } p \mid q
\end{aligned}
$$

So using the minimal polynomial of $A$ over $I F_{p}$ we can determine all $p$-ranks except

$$
r k_{p}(A+d I) \text { for } p \mid q \text {. }
$$

This result also follows directly from Godsil's theorem since for a Hamming graph $H(d, q)$ we have $|\operatorname{det} P|^{2}=q^{d(d+1)}$.

According to Corollary 3.2 for $p$ not dividing $q$ the $p$-rank of $H(d, q)-c I$, considering its spectrum, is as small as possible for any integral $c$. So if $A$ is the adjacency matrix of some graph cospectral with the Hamming graph $H(d, q)$ and $p$ is a prime not dividing q, then $r k_{p}(A-c I) \geq r k_{p}(H(d, q)-c I)$ for any $c$. If $d=3$ and $q$ is odd we can also show the converse, namely that the distance-regularity follows from the minimality of some 2-rank:

Theorem 3.3 Let for some odd $q, \Gamma$ be a graph with the same spectrum as $H(3, q)$ and let $A$ be its adjacency matix. If $r k_{2}(A+I)=1+3(q-1)^{2}$, then $\Gamma$ is isomorphic to $H(3, q)$.

Proof: It follows from the spectrum that $\Gamma$ is regular of degree $3(q-1)$ and that two adjacent vertices have the right number $(q-2)$ common neighbors (see [7]). Furthermore 
$r k_{2}(A)=3(q-1)+(q-1)^{3}$, so modulo $2 \Gamma$ has minimal polynomial $x^{2}+x$. So $A^{2} \equiv A$ $(\bmod 2)$ and two vertices at distance 2 have at least 2 common neighbors, implying that each vertex has at most $3(q-1)^{2}$ vertices at distance 2. It is proved in Haemers [7] that each vertex has at least this many vertices at distance 2 and that if equality holds for each vertex, the graph is distance-regular. Since $H(3, q)$ is the unique distance-regular graph with this spectrum, the result follows.

This theorem also proves the characterisation of $H(3,3)$ by its spectrum and the minimality of the 2-rank of $A+I$ that we saw in Example 1.1.

We will now determine the $p$-rank of the matrix $H(d, q)+d I$ for every prime number $p$ dividing $q$ and the corresponding 2-rank for the Doob graphs. Using the fact that these graphs are direct products of complete graphs and/or Shrikhande graphs, a recurrence relation can be derived for the considered $p$-rank. We will denote the matrix $H(d, q)+d I$ by $B(d, q)$, the adjacency matrix of the Doob graph $D(n, m)$ by $D(n, m)$ and we define $\underline{r}_{d}$ to be the vector of length $p$ with the $k$-th coefficient equal to $r k_{p} B(d, q)^{k-1}$. For two integral matrices $M$ and $N$, the expression $M \sim_{p} N$ will mean that, considered as matrices over $I F_{p}, M$ and $N$ have the same rank.

Theorem 3.4 Let $p$ be a prime dividing $q$, then

$$
\underline{r}_{d}=Q^{d} \underline{r}_{0}
$$

where

$$
\underline{r}_{0}=(1,0,0, \ldots, 0)^{T} \text { and } Q=\left(\begin{array}{cccccc}
q & 0 & 0 & \ldots & \ldots & 0 \\
1 & q-2 & 1 & 0 & \ldots & 0 \\
0 & 1 & q-2 & 1 & \ddots & \vdots \\
\vdots & \ddots & \ddots & \ddots & \ddots & 0 \\
\vdots & & \ddots & \ddots & \ddots & 1 \\
0 & \ldots & \ldots & 0 & 1 & q-2
\end{array}\right)
$$

If $p=2$, then

$$
\underline{r}_{d}=\left(\begin{array}{c}
q^{d} \\
\frac{1}{2}\left(q^{d}-(q-2)^{d}\right)
\end{array}\right)
$$

If $p=3$, then

$$
\begin{gathered}
\underline{r}_{d}=\left(\begin{array}{c}
q^{d} \\
\frac{2}{3} q^{d}-\frac{1}{2}(q-1)^{d}-\frac{1}{6}(q-3)^{d} \\
\frac{1}{3} q^{d}-\frac{1}{2}(q-1)^{d}+\frac{1}{6}(q-3)^{d}
\end{array}\right) \\
r k_{2}(D(n, m)+n I)=\frac{1}{2}\left(4^{n+2 m}-2^{n+2 m}\right)
\end{gathered}
$$


Proof: Let $p$ be a prime dividing $q$, let $M$ be an integral matrix and $l \neq \equiv 0(\bmod p)$ an integer, then it follows from a straightforward reduction (cf. [10]) that

$$
\left(I_{q} \otimes M^{k}\right)+l\left(J_{q} \otimes M^{k-1}\right) \sim_{p} \operatorname{diag}\left(M^{k-1},\left(M^{k}\right)^{q-2}, M^{k+1}\right) .
$$

Because $B(d, q)=H(d, q)+d I$, we have the following recurrence relation for $B(d, q)$ :

$$
B(d, q)=I_{q} \otimes B(d-1, q)+J_{q} \otimes I
$$

from which we derive that

$$
B(d, q)^{k} \equiv I_{q} \otimes B(d-1, q)^{k}+k J_{q} \otimes B(d-1, q)^{k-1} \quad(\bmod p)
$$

if $p \mid q$. Now $B(0, q)=(0)$, so by induction $B(d, q)^{p} \equiv 0(\bmod p)$. From (5) and (6) we get the following recurrence relation for the $p$-rank of powers of the matrix $B(d, q)$ :

$$
r k_{p} B(d, q)^{k}=r k_{p} B(d-1, q)^{k-1}+(q-2) r k_{p} B(d-1, q)^{k}+r k_{p} B(d-1, q)^{k+1}
$$

for $k=1, \ldots, p-1$ and with $p \mid q$. Together with the obvious relations

$$
\begin{array}{ll}
r k_{p} B(0, q)^{k}=0 & \text { for } k>0 \\
r k_{p} B(d, q)^{p}=0 & \text { for } d \geq 0 \\
r k_{p} B(d, q)^{0}=q^{d} & \text { for } d \geq 0
\end{array}
$$

these determine the $p$-ranks of the powers of $B(d, q)$ completely. The above relations can be rewritten as

$$
\underline{r}_{d}=\underline{r}_{d-1}
$$

with

$$
\underline{r}_{0}=(1,0,0, \ldots, 0)^{T} \text { and } Q=\left(\begin{array}{cccccc}
q & 0 & 0 & \ldots & \ldots & 0 \\
1 & q-2 & 1 & 0 & \ldots & 0 \\
0 & 1 & q-2 & 1 & \ddots & \vdots \\
\vdots & \ddots & \ddots & \ddots & \ddots & 0 \\
\vdots & & \ddots & \ddots & \ddots & 1 \\
0 & \ldots & \ldots & 0 & 1 & q-2
\end{array}\right)
$$

So

$$
\underline{r}_{d}=Q^{d} \underline{r}_{0}
$$


For $p \neq 2,3$ this will be the most useful expression we get for the $p$-rank of $H(d, q)+d I$ and its powers. For $p=2,3$ we can derive explicit formulas for the $p$-ranks we are looking for by diagonalizing the matrix $Q$. Namely if $p=2$ then

$$
Q=\left(\begin{array}{cc}
q & 0 \\
1 & q-2
\end{array}\right)=\left(\begin{array}{ll}
2 & 0 \\
1 & 1
\end{array}\right)\left(\begin{array}{cc}
q & 0 \\
0 & q-2
\end{array}\right)\left(\begin{array}{ll}
2 & 0 \\
1 & 1
\end{array}\right)^{-1}
$$

and if $p=3$ then

$$
Q=\left(\begin{array}{ccc}
q & 0 & 0 \\
1 & q-2 & 1 \\
0 & 1 & q-2
\end{array}\right)=\left(\begin{array}{ccc}
3 & 0 & 0 \\
2 & 1 & 1 \\
1 & 1 & -1
\end{array}\right)\left(\begin{array}{ccc}
q & 0 & 0 \\
0 & q-1 & 0 \\
0 & 0 & q-3
\end{array}\right)\left(\begin{array}{ccc}
3 & 0 & 0 \\
2 & 1 & 1 \\
1 & 1 & -1
\end{array}\right)^{-1}
$$

yielding (3) and (4).

Let $S h$ be the adjacency matrix of the Shrikhande graph and $M$ an integral $s \times s$-matrix, then

$$
\left(I_{16} \otimes M\right)+\left(S h \otimes I_{s}\right) \sim_{2} \operatorname{diag}\left(\left(I_{s}\right)^{6},(M)^{4},\left(M^{2}\right)^{6}\right)
$$

The proof is again a straightforward reduction and is omitted here. The recursion formula can also be obtained by using (5) and the fact that the matrix $S h$ is obtained from $H(2,4)$ by permuting the rows.

Let $S h(m)$ denote the adjacency matrix of the direct product of $m$ copies of a Shrikhande graph, then it satisfies the following recurrence relation:

$$
\operatorname{Sh}(m)=I_{16} \otimes S h(m-1)+S h \otimes I_{16^{m-1}} .
$$

So by induction $\operatorname{Sh}(m)^{2} \equiv 0(\bmod 2)$. Using (7) we get that

$$
r k_{2} \operatorname{Sh}(m)=6 \cdot 16^{m-1}+4 \cdot r k_{2} \operatorname{Sh}(m-1),
$$

which yields:

$$
r k_{2} \operatorname{Sh}(m)=\frac{1}{2}\left(16^{m}-4^{m}\right)=r k_{2} B(2 m, 4)
$$

Now by induction on $n$ (or $m$ ) using (5) and (8) (or (7) and (3)) it follows that

$$
r k_{2}(D(n, m)+n I)=\frac{1}{2}\left(4^{n+2 m}-2^{n+2 m}\right) .
$$

Remark 3.1 Note that

$$
r k_{2}(D(n, m)+n I)=\frac{1}{2}\left(4^{n+2 m}-2^{n+2 m}\right)=r k_{2}(H(n+2 m, 4)+n I) .
$$


So each $p$-rank of a Doob graph is the same as the one for the Hamming graph with the same parameters as this Doob graph.

Remark 3.2 It follows from the recurrence relations for $B(d, q)$ and $D(n, m)$ that for $p$ dividing $q$ we have $\mathbf{1} \in\langle H(d, q)+d I\rangle_{p}$ and $\mathbf{1} \in\langle D(n, m)+n I\rangle_{2}$, so $r k_{p}(H(d, q)+d I+b J)$ and $r k_{2}(D(n, m)+n I+b J)$ (for integral $\left.b\right)$ are independent of $b$.

Remark 3.3 It is possible to find for every $p$ and $k \in\{1,2, \ldots, p-1\}$ coefficients $\alpha_{1}, \alpha_{2}, \ldots, \alpha_{p}$ depending on $p$ and $k$ only, such that

$$
r k_{p} B(d, q)^{k}=\alpha_{1} q^{d}+\alpha_{2}\left(q-2+x_{1}\right)^{d}+\cdots+\alpha_{p}\left(q-2+x_{p-1}\right)^{d}
$$

with $x_{1}, x_{2}, \ldots, x_{p-1}$ zero's of the polynomial $S_{p-1}(x)$ defined by $S_{n}(x)=\operatorname{det}\left(S_{n}+x I_{n}\right)$, where $S_{n}$ is the $n \times n$ matrix with one's on the codiagonals and zero's elsewhere. One can prove that the roots $x_{i}$ lie symmetric with respect to 0 and $\left|x_{i}\right|<2$ for $i=1,2, \ldots, n$.

\section{Some other distance-regular graphs}

\subsection{Johnson graphs}

The Johnson graph $J(n, k)$ is the graph with vertex set the $k$-subsets of a set with $n$ elements, two vertices being adjacent if they have an intersection of size $k-1$. The Johnson graph $J(n, k)$ has $\left(\begin{array}{l}n \\ k\end{array}\right)$ vertices, diameter $d=\min \{k, n-k\}$ and is distance-regular with intersection numbers $a_{i}=(n-2 i) i, b_{i}=(k-i)(n-k-i), c_{i}=i^{2}$. It has eigenvalues $\theta_{i}=k(n-k)-i(n+1-i)=k(n-k)-\left(\frac{n+1}{2}\right)^{2}+\left(i-\frac{n+1}{2}\right)^{2}$ with multiplicities $f_{i}=\left(\begin{array}{c}n \\ i\end{array}\right)-\left(\begin{array}{c}n \\ i-1\end{array}\right)(0 \leq i \leq d)$.

Let $p$ be a prime and let $A$ be the adjacency matrix of a distance-regular graph with the same parameters as $J(n, k)$. Then by Lemma 2.2, A modulo $p$ has minimal polynomial

$$
\begin{aligned}
& (x+k)^{2} \\
& \text { if } p=2 \text { and } n \text { even, } \\
& x(x+1) \\
& \text { if } p=2 \text { and } n \text { odd, } \\
& \prod_{i=0}^{d}\left(x-\theta_{i}\right) \quad(\bmod p) \\
& \text { if } p \text { is odd and } p>d \text {, } \\
& \prod_{i=0}^{p-1}\left(x-k(n-k)+\left(\frac{n+1}{2}\right)^{2}-i^{2}\right) \text { if } p \text { is odd and } p \leq d \text {. }
\end{aligned}
$$

So we have

$$
r k_{p}\left((A-c I)^{2}\right)=\sum_{\theta_{i} \neq c(\bmod p)} f_{i}
$$


for all prime numbers $p$ and integral $c$, and in fact

$$
r k_{p}(A-c I)=\sum_{\theta_{i} \neq c(\bmod p)} f_{i}
$$

for all prime numbers $p$ and integers $c$, except possibly for

$$
\begin{array}{ll}
r k_{2}(A+k I) & \text { if } n \text { is even, } \\
r k_{p}\left(A-\left(k(n-k)-\left(\frac{n+1}{2}\right)^{2}+c\right) I\right) & \begin{array}{l}
\text { with } p \text { odd and } c \text { is a } \\
\text { non-zero square }(\bmod p) .
\end{array}
\end{array}
$$

Concerning these cases we can determine only a few more $p$-ranks. From now on we will denote the adjacency matrix of $J(n, k)$ by $J(n, k)$ as well. If $n$ is even then $r k_{2}(J(n, k)+$ $k I)=r k_{2}(J(n-1, k)+k I)$ since the sum of all rows of $J(n, k)+k I$ corresponding to the $k$-subsets that contain some fixed $k-1$-set is equal to the zero vector modulo 2 . From this we get the following values for the 2-ranks of $J(n, k)$ which were also found by R. Riebeek (personal communication):

$$
\begin{aligned}
r k_{2}(J(n, k)+k I)= & \left(\begin{array}{l}
n-2 \\
k-1
\end{array}\right) \text { if } n \text { is even, } \\
& \left(\begin{array}{l}
n-1 \\
k-1
\end{array}\right) \text { if } n \text { is odd, } \\
r k_{2}(J(n, k)+(k+1) I)= & \left(\begin{array}{l}
n \\
k
\end{array}\right) \quad \text { if } n \text { is even, } \\
& \left(\begin{array}{c}
n-1 \\
k
\end{array}\right) \text { if } n \text { is odd. }
\end{aligned}
$$

Some other results follow from the work of Wilson (see [12]) who, for given integers $t, k$ and $n$, determined the $p$-ranks of the $\left(\begin{array}{c}n \\ t\end{array}\right)$ by $\left(\begin{array}{c}n \\ k\end{array}\right)$ matrix $N_{t, k}(n)$ (or simply $N_{t, k}$ ) of 0 's and 1's, the rows of which are indexed by the $t$-subsets $T$ of an $n$-set $X$, whose columns are indexed by the $k$-subsets $K$ of the same set $X$, and where the entry $N_{t, k}(T, K)$ in row $T$ and column $K$ is 1 if $T \subseteq K$ and is 0 otherwise. He proved the following theorem:

Theorem 4.1 (Wilson) (cf. [12]) For $t \leq \min \{k, n-k\}$, the rank of $N_{t, k}$ modulo a prime $p$ is

$$
\sum\left(\begin{array}{l}
n \\
i
\end{array}\right)-\left(\begin{array}{c}
n \\
i-1
\end{array}\right)
$$

where the sum is extended over those indices $i$ such that $p$ does not divide the binomial coefficient

$$
\left(\begin{array}{c}
k-i \\
t-i
\end{array}\right)
$$


Now by definition $J(n, k)+k I=N_{k-1, k}^{T} N_{k-1, k}$. By Wilson's theorem

$$
r k_{p}\left(N_{k-1, k}\right)=\sum_{\substack{i=0 \\
i \neq k}}^{k-1}\left(\begin{array}{l}
n \\
i
\end{array}\right)-\left(\begin{array}{c}
n \\
i-1
\end{array}\right),
$$

so for instance $r k_{p}\left(N_{k-1, k}\right)=\left(\begin{array}{c}n \\ k-1\end{array}\right)$ if $p>k, r k_{p}\left(N_{p-1, p}\right)=\left(\begin{array}{c}n \\ p-1\end{array}\right)-1$ and $r k_{3}\left(N_{3,4}\right)=$ $\left(\begin{array}{l}n \\ 3\end{array}\right)-(n-1)$ from which, after considering the kernel of $N_{k-1, k}$, the following results follow:

$$
\begin{aligned}
r k_{p}(J(n, k)+k I)= & \left(\begin{array}{c}
n \\
k-1
\end{array}\right) \quad \text { if } p>k \\
r k_{p}(J(n, p)+p I)= & \left(\begin{array}{c}
n \\
p-1
\end{array}\right)-2 \quad \text { if } p \text { divides }\left(\begin{array}{c}
n \\
p-1
\end{array}\right), \\
& \left(\begin{array}{c}
n \\
p-1
\end{array}\right)-1 \quad \text { if } p \text { does not divide }\left(\begin{array}{c}
n \\
p-1
\end{array}\right), \\
r k_{3}(J(n, 4)+4 I)= & \left(\begin{array}{l}
n \\
3
\end{array}\right)-2(n-1)+1 \quad \text { if } n \equiv 0 \quad(\bmod 3), \\
& \left(\begin{array}{l}
n \\
3
\end{array}\right)-(n-1) \quad \text { if } n \equiv 1 \quad(\bmod 3), \\
& \left(\begin{array}{l}
n \\
3
\end{array}\right)-2(n-1) \quad \text { if } n \equiv 2 \quad(\bmod 3) .
\end{aligned}
$$

We omit the proof because these results cover only a few of the remaining cases and its proof is technical and straightforward. Determining all relevant $p$-ranks for the Johnson graphs seems to be a difficult problem.

\subsection{GQ minus a spread}

Let $G Q(s, t)$ be a generalized quadrangle with point set $\mathcal{P}$ and line set $\mathcal{L}$. A spread is a collection of lines partitioning the point set. Let $\mathcal{S}$ be a spread of $G Q(s, t)$, then (cf. [2] Section 12.5 or [1]) the collinearity graph $\Gamma$ of $(\mathcal{P}, \mathcal{L} \backslash \mathcal{S})$ is distance-regular of diameter 3 , with $v=(s+1)(s t+1)$ vertices, spectrum $s t^{1},-1^{s t=m_{2}}, s^{s t(s t+1) /(s+t)=m_{1}},-t^{s^{2}(s t+1) /(s+t)=m_{3}}$ and intersection array $\{s t, s(t-1), 1 ; 1, t-1, s t\}$, an antipodal $(s+1)$-cover of the complete graph $K_{s t+1}$. More generally, given a strongly regular graph $\Delta$ with parameters $(v, k, \lambda, \mu)=$ $((s+1)(s t+1), s(t+1), s-1, t+1)$ such that there is a partition $\mathcal{S}$ of its point set into $(s+1)$-cliques, the partial graph $\Gamma$ obtained by deleting the edges contained in the members of $\mathcal{S}$ is distance-regular of diameter 3 with intersection array as given above. Conversely, any graph $\Gamma$ with these parameters arises in this way.

Let $G$ be a graph with the same spectrum as $\Gamma$ and let $A$ be its adjacency matrix, then the only $p$-ranks that are not necessarily determined by the spectrum are

$$
\begin{array}{lll}
r k_{p}(A+I) & \text { for } p \mid(s+1) & \text { or } \\
r k_{p}(A+t I) & \text { for } p \mid(s+t) &
\end{array}
$$

If furthermore $G$ is distance-regular we can say more. 
Theorem 4.2 Let $\Gamma$ be a distance-regular graph with adjacency matrix A, intersection array $\{s t, s(t-1), 1 ; 1, t-1, s t\}$ and spectrum $s t^{1}, s^{m_{1}},-1^{m_{2}},-t^{m_{3}}$. Let $\mathcal{S}$ be the partition of the point set into the st +1 antipodal $(s+1)$-tuples and let $\Delta$ be the strongly regular graph with adjacency matrix $B$, parameters $(v, k, \lambda, \mu)=((s+1)(s t+1), s(t+1), s-1, t+1)$ and spectrum $s(t+1)^{1},(s-1)^{m_{1}+m_{2}},-(t+1)^{m_{3}}$ obtained from $\Gamma$ by adding the edges between antipodal pairs of vertices.

If $p$ divides $t-1$, but not $s+1$, then

$$
r k_{p}(A+I)=1+m_{1} .
$$

If $p$ divides $s+1$, but not $t-1$, then

$$
r k_{p}(A+I)=1+m_{2}+m_{3} .
$$

If $p$ divides $s+t$, but not $s+1$, then

$$
r k_{p}(A+t I)=r k_{p}(B+(t+1) I)+s t-\epsilon,
$$

where $\epsilon=1$ if $p$ divides $t$ and $\epsilon=0$ otherwise.

Proof: Let $p$ be a prime dividing $t-1$, then since $\Gamma$ is distance-regular it follows from Lemma 2.2 that $(x+1)(x-s)$ is the minimal polynomial of $A$ over $I F_{p}$, so if $p$ does not divide $s+1$, then $r k_{p}(A+I)=1+m_{1}$ and $r k_{p}(A-s I)=m_{2}+m_{3}$.

Let $p$ be a prime dividing $s+1$, but not $t-1$. Denote the $s t+1$ antipodal $(s+1)$-tuples of $\Gamma$ as well as their characteristic vectors by $l_{1}, l_{2}, \ldots, l_{s t+1}$ and let $S\left(=I_{s t+1} \otimes J_{s+1}\right)$ be the matrix for which $S_{i j}=1$ if $i=j$ or if $i$ and $j$ are antipodal in $\Gamma$ and $S_{i j}=0$ otherwise, then $B+I=A+S$ and

$$
\langle A+I\rangle_{p}+\left\langle l_{1}, \ldots, l_{s t+1}\right\rangle_{p}=\langle B+2 I\rangle_{p}+\left\langle l_{1}, \ldots, l_{s t+1}\right\rangle_{p} .
$$

\section{Claim 1}

$$
l_{i} \in\langle A+I\rangle_{p} \quad \text { for } i=1,2, \ldots, s t+1
$$

Summing all rows of $A+I$ gives $(s t+1) \mathbf{1} \equiv(1-t) \mathbf{1}(\bmod p)$, so the all-one vector is in $\langle A+I\rangle_{p}$. Since $\Delta$ is strongly regular with the given spectrum, we have $(B+2 I)(B+$ $(t+1) I) \equiv(t+1) J(\bmod p)$. Furthermore we have that $S(B+2 I) \equiv J(\bmod p)$ and $S^{2} \equiv O(\bmod p)$, so $(A+t I)(A+I)=(B+(t+1) I-S)(B+2 I-S)=(B+(t+1) I)$ $(B+2 I)-S(2 B+(t+3) I)+S^{2} \equiv(t+1) J-2 J-(t-1) S=(t-1)(J-S)(\bmod p)$, so $1-l_{i}$ and hence $l_{i}$ is in $\langle A+I\rangle_{p}$.

\section{Claim 2}

$$
\langle B+2 I\rangle_{p} \cap\left\langle l_{1}, \ldots, l_{s t+1}\right\rangle_{p}=\langle\mathbf{1}\rangle_{p}
$$

Note that $\left\langle l_{1}, \ldots, l_{s t+1}\right\rangle_{p}=\left\langle\mathbf{1}, l_{1}-l_{2}, \ldots, l_{1}-l_{s t+1}\right\rangle_{p}$ since $\mathbf{1}+\sum_{i=1}^{s t+1}\left(l_{1}-l_{i}\right)=\mathbf{1}-$ $\sum l_{i}+(s t+1) l_{1} \equiv(1-t) l_{1}(\bmod p)$. Now $l_{i}(B+2 I)=\mathbf{1}$, so $l_{i}-l_{j} \in \operatorname{ker}_{p}(B+2 I)$ for all $i, j=1,2, \ldots, s t+1$. Since $B$ (over $I F_{p}$ ) has minimal polynomial $(x+t+1)^{2}(x+2)$ we have $\operatorname{ker}_{p}(B+2 I) \cap\langle B+2 I\rangle_{p}=\{0\}$ and the claim follows. 
Now

$$
\begin{aligned}
r k_{p}(A+I)= & \operatorname{dim}\left(\langle A+I\rangle_{p}+\left\langle l_{1}, \ldots, l_{s t+1}\right\rangle_{p}\right)=\operatorname{dim}\left(\langle B+2 I\rangle_{p}\right. \\
& \left.+\left\langle l_{1}, \ldots, l_{s t+1}\right\rangle_{p}\right)=r k_{p}(B+2 I)+s t=1+m_{2}+m_{3} .
\end{aligned}
$$

Let $p$ be a prime dividing $s+t$ but not $s+1$, then again

$$
\langle A+t I\rangle_{p}+\left\langle l_{1}, \ldots, l_{s t+1}\right\rangle_{p}=\langle B+(t+1) I\rangle_{p}+\left\langle l_{1}, \ldots, l_{s t+1}\right\rangle_{p}
$$

Since $l_{i}(A+t I)=\mathbf{1}+(t-1) l_{i}$ the left hand side of (9) has dimension $r k_{p}(A+t I)$ or $r k_{p}(A+t I)+1$ depending on whether $\mathbf{1} \in\langle A+t I\rangle_{p}$ or not. Summing the rows of $A+t I$ yields $(s+1) t \mathbf{1}$, so $\mathbf{1} \in\langle A+t I\rangle_{p}$ if $p \nmid t$. If on the other hand $p \mid t$, then $\langle A+t I\rangle_{p} \subset \mathbf{1}^{\perp}$, but $\mathbf{1 1}^{T}=(s+1)(s t+1) \equiv(s+1) \not \equiv 0 \quad(\bmod p)$, so $\mathbf{1} \notin\langle A+t I\rangle_{p}$.

For each $l_{i}$ we have that $l_{i}(B+(t+1) I)=\mathbf{1}$, so $l_{i}-l_{j} \in \operatorname{ker}_{p}(B+(t+1) I)$ for any two $(s+1)$-tuples of $\mathcal{S}$. Let $\chi:=\sum_{i=1}^{s t+1} \alpha_{i} l_{i}$ be a vector from $\left\langle l_{1}, \ldots l_{s t+1}\right\rangle_{p}$ and suppose that $\chi \in\langle B+(t+1) I\rangle_{p}$. Then, since $l_{i}-l_{j} \in \operatorname{ker}_{p}(B+(t+1) I)$ and $B$ is symmetric, we must have that $l_{i} \chi^{T}$ is constant for all $l_{i}$. Now $l_{i} \chi^{T}=\alpha_{i}(s+1)$ and $p$ does not divide $s+1$, so $\chi \in\langle\mathbf{1}\rangle_{p}$. Since $\mathbf{1} \in\langle B+(t+1) I\rangle_{p}$ the dimension of the right hand side of (9) is equal to $r k_{p}(B+(t+1) I)+s t$.

Note that all $p$-ranks covered bij Theorem 4.2 are determined by the graph $\Delta$ alone and do not depend on the particular spread $\mathcal{S}$ that is deleted from $\Delta$. So the only $p$-ranks that can actually depend on the particular spread that is deleted are

$$
r k_{p}(A+t I) \text { for } p \text { dividing } s+t \text { as well as } t-1 \text {. }
$$

The next example illustrates that indeed we can have different $p$-ranks for graphs obtained from the same $G Q$ by deleting different spreads.

Example 4.1 Up to isomorphism there are two distance-regular graphs on 27 vertices with intersection array $\{8,6,1 ; 1,3,8\}$ (cf. [1]). Both are the collinearity graph of the unique $G Q(2,4)$ minus a spread. This $G Q$ possesses exactly two non-isomorphic spreads (see [4]). In the dual $G Q(4,2)$ one spread corresponds to a 'plane-ovoid' and the other to a 'tripod'. The considered graphs have spectrum $8^{1}, 2^{12},-1^{8},-4^{6}$, so if $A$ is the adjacency matrix of a graph $\Gamma$ with this spectrum, $r k_{2}(A)$ and $r k_{3}(A+I)$ are in general not determined by this spectrum. If $\Gamma$ is $G Q(2,4)$ minus a spread, then $r k_{2}(A)=r k_{2}(B+I)+s t-1=14$, where $B$ is the adjacency matrix of $G Q(2,4)$ for which $r k_{2}(B+I)=7$. In [8] all graphs with spectrum $8^{1}, 2^{12},-1^{8},-4^{6}$ are determined. There are 13 of these. Their ranks are listed in Table 3.

\subsection{Square 2-designs}

Any connected bipartite graph $\Gamma$ is the incidence graph of a design $(X, \mathcal{B})$. It can be found in [2] (Section 1.6) that $\Gamma$ is a (bipartite) distance-regular graph of diameter 3 if and only if $(X, \mathcal{B})$ is a square 2 -design. If the square 2-design has parameters $2-(w, k, \mu)$, then $\Gamma$ has 
Table 3. The relevant $p$-ranks of all graphs cospectral with $G Q(2,4)$ minus a spread.

\begin{tabular}{|c|c|c|c|c|c|}
\hline & $|\operatorname{Aut}(\Gamma)|$ & $r k_{2}(A)$ & $r k_{3}(A$ & $+I)$ & \\
\hline 1 & 4 & 120 & 14 & - & \\
\hline 2 & 36 & 120 & 14 & - & \\
\hline 3 & 2 & 140 & 14 & - & \\
\hline 4 & 12 & 140 & 14 & - & \\
\hline 5 & 12 & 140 & 12 & - & \\
\hline 6 & 16 & 140 & 14 & - & \\
\hline 7 & 4 & 120 & 14 & - & \\
\hline 8 & 12 & 120 & 14 & - & \\
\hline 9 & 16 & $10 \quad 0$ & 14 & - & \\
\hline 10 & 24 & 120 & 14 & - & \\
\hline 11 & 1296 & 80 & 14 & - & $\Gamma_{3}$ of $H(3,3)$ \\
\hline 12 & 1296 & 140 & 10 & - & $G Q(2,4)$ minus 'plane-ovoid'-spread \\
\hline 13 & 324 & 140 & 12 & - & $G Q(2,4)$ minus 'tripod'-spread \\
\hline
\end{tabular}

$2 w$ vertices, intersection array $\{k, k-1, k-\mu ; 1, \mu, k\}$ and spectrum $\pm k^{1}, \pm \sqrt{k-\mu}^{w-1}$. It is proved in [7] that any graph with this spectrum is distance-regular and hence the incidence graph of a square 2-design.

Let $\Gamma$ be a graph with spectrum $\pm k^{1}, \pm \sqrt{k-\mu}^{w-1}$. Let $A$ be its adjacency matrix and $N$ the incidence matrix of the corresponding square 2-design with parameters 2- $(w, k, \mu)$ (so $k(k-1)=\mu(w-1))$, then the following $p$-ranks are still open:

$$
\begin{array}{ll}
r k_{p}(A+k I) & \text { for } p \mid k^{2}-k+\mu=\mu w \\
r k_{2}(A+(k-\mu) I) & \\
r k_{p}(A) & \text { for odd } p \text { for which } p^{2} \mid(k-\mu)
\end{array}
$$

Notice that

$$
A=\left(\begin{array}{cc}
O & N \\
N^{T} & O
\end{array}\right) .
$$

Suppose that $p \mid k^{2}-k+\mu$ and $p \nmid k$ then $r k_{p}(A+k I)=r k_{p} \operatorname{diag}\left(I_{w}, N^{T} N-k^{2} I\right)=$ $w+r k_{p}\left(\mu J+\left(k^{2}-k+\mu\right) I\right)=w+r k_{p}(\mu J)$. Similarly, if $k-\mu$ is odd then $r k_{2}(A+I)=$ $w+r k_{2}(\mu J)$. If $2 \|(k-\mu)$ then $r k_{2}(N)=\frac{w+1}{2}$ and hence $r k_{2}(A)=w+1$ if $\mu$ is odd and $r k_{2}(N)=\frac{w-1}{2}$ and hence $r k_{2}(A)=w-1$ if $\mu$ is even. The $p$-ranks that remain are:

$$
r k_{p}(A)=2 r k_{p}(N) \quad \text { for } p^{2} \mid(k-\mu) .
$$

The $p$-ranks $r k_{p}(N)$ with $p^{2} \mid(k-\mu)$ are precisely those that are not determined by the parameters of the design, see [9]. 


\subsection{Taylor graphs}

A distance-regular graph with intersection array $\{k, \mu, 1 ; 1, \mu, k\}$ is called a Taylor graph. For a Taylor graph the number of vertices at distance 2 and 3 from a point is $k$ and 1 respectively, so $\Gamma$ is an antipodal double cover of $K_{k+1}$ and has spectrum $k^{1},-1^{k}, \lambda_{2}^{m_{2}}, \lambda_{3}^{m_{3}}$, with $\lambda_{2}+\lambda_{3}=k-1-2 \mu, \lambda_{2} \lambda_{3}=-k, m_{2}=\frac{-\lambda_{3}}{\lambda_{2}-\lambda_{3}}(k+1)$, and $m_{3}=\frac{\lambda_{2}}{\lambda_{2}-\lambda_{3}}(k+1)$.

Let $A$ be the adjacency matrix of a graph $\Gamma$ with this spectrum, then the following $p$-ranks are not necessarily determined by the spectrum:

$$
\begin{array}{ll}
r k_{p}(A+I) & \text { for } p \mid 2 \mu \\
r k_{p}\left(A-\frac{1}{2}(k-1-2 \mu) I\right) & \text { for odd } p \text { with } p^{2} \mid(k-1-2 \mu)^{2}+4 k
\end{array}
$$

If $\Gamma$ is distance-regular and $p \mid \mu$, then by Lemma $2.2(x+1)(x-k)$ is the minimal polynomial of $A$ modulo $p$, so if $p \mid \mu$ but $p \nmid k+1$, the geometric multiplicities of the eigenvalues -1 and $k$ are equal to their algebraic multiplicities.

Suppose that $\Gamma$ is a Taylor graph, let $x$ be a vertex of $\Gamma$ and let $B_{x}$ be the adjacency matrix of $\overline{\Gamma(x)}$, the complement of the subgraph of $\Gamma$ induced by the neighbors of $x$. The graph $\overline{\Gamma(x)}$ is strongly regular with parameters $\left(k, \mu, \frac{1}{2}(-k-1+3 \mu), \frac{1}{2} \mu\right)$ (so $\mu$ is even and $k$ is odd) and has eigenvalues $\mu$ and $r, s=-\frac{1}{4}(k+1-2 \mu) \pm \frac{1}{4} \sqrt{(k-1-2 \mu)^{2}+4 k}$. Let

$$
B_{x}^{\prime}:=\left(\begin{array}{c|c}
0 & \mathbf{0} \\
\hline \mathbf{0}^{T} & B_{x}
\end{array}\right),
$$

then

$$
A=\left(\begin{array}{c|c}
J-I-B_{x}^{\prime} & B_{x}^{\prime} \\
\hline B_{x}^{\prime} & J-I-B_{x}^{\prime}
\end{array}\right)
$$

and it follows that the $p$-ranks of the Taylor graphs that are not yet determined by the spectrum, can be expressed in terms of the $p$-ranks of $\overline{\Gamma(x)}$ :

Theorem 4.3 Let $A$ and $B_{x}$ be as before, then for any prime $p$

$$
r k_{p}(A+I)=2+r k_{p}\left(B_{x}\right)
$$

If $p$ is an odd prime for which $p^{2} \mid(k-1-2 \mu)^{2}+4 k$ and $p$ does not divide $\mu$, then

$$
r k_{p}\left(A-\frac{1}{2}(k-1-2 \mu) I\right)=k+\epsilon+r k_{p}\left(B_{x}+\frac{1}{4}(k+1-2 \mu) I\right)
$$

where $\epsilon=0$ if $p \mid k$ and $\epsilon=1$ otherwise. 
Note that $r k_{p}\left(B_{x}\right)$ is not necessarily determined by its spectrum if $p$ divides both $\frac{1}{2} \mu$ and $\frac{1}{2}(k+1)$, since $(x-r)(x-s) \equiv x^{2} \quad(\bmod p)$ in these cases. For an odd prime $p$ with $p^{2} \mid(k-1-2 \mu)^{2}+4 k$ the rank $\left.r k_{p}\left(B_{x}+\frac{1}{4}(k+1-2 \mu) I\right)\right)$ is in general not determined by the spectrum of $B_{x}$.

Proof: The first identity follows straightforwardly from the fact that $\mathbf{1} \notin\left\langle B_{x}^{\prime}\right\rangle_{p}$. Now suppose we are in the second case, so $p$ is odd, $p^{2}$ divides $(k-1-2 \mu)^{2}+4 k$ and $p$ does not divide $\mu$. Then

$$
\begin{aligned}
A & -\frac{1}{2}(k-1-2 \mu) I \\
& \sim_{p}\left(\begin{array}{c|c}
2 J-2 B_{x}^{\prime}-(k+1-2 \mu) I & 2 B_{x}^{\prime} \\
\hline 2 B_{x}^{\prime} & 2 J-2 B_{x}^{\prime}-(k+1-2 \mu) I
\end{array}\right) \\
& \sim_{p}\left(\begin{array}{c|c}
2 J-(k+1-2 \mu) I & 2 B_{x}^{\prime} \\
\hline 2 J-(k+1-2 \mu) I & 2 J-2 B_{x}^{\prime}-(k+1-2 \mu) I
\end{array}\right) \\
& \sim_{p}\left(\begin{array}{c|c}
2 J-(k+1-2 \mu) I & 2 B_{x}^{\prime} \\
\hline O & 2 J-4 B_{x}^{\prime}-(k+1-2 \mu) I
\end{array}\right) \\
& \sim_{p}\left(\begin{array}{c|c}
2 J-(k+1-2 \mu) I & O \\
\hline O & 2 J-4 B_{x}^{\prime}-(k+1-2 \mu) I
\end{array}\right) .
\end{aligned}
$$

So

$$
\begin{aligned}
r k_{p}\left(A-\frac{1}{2}(k-1-2 \mu) I\right)= & r k_{p}(2 J-(k+1-2 \mu) I) \\
& +r k_{p}\left(2 J-4 B_{x}^{\prime}-(k+1-2 \mu) I\right) .
\end{aligned}
$$

Since $p$ does not divide $(k+1-2 \mu)$ (this would imply that $p$ divides $\mu$, a contradiction) $r k_{p}(2 J-(k+1-2 \mu) I)$ is equal to either $k+1$ or $k$. We are in the second case if and only if $p \mid(k+1+2 \mu)$, which is the case if and only if $p \mid k$.

In order to prove that

$$
r k_{p}\left(2 J-4 B_{x}^{\prime}-(k+1-2 \mu) I\right)=r k_{p}\left(B_{x}+\frac{1}{4}(k+1-2 \mu) I\right)
$$

we consider the cases $p \mid k$ and $p \nmid k$ separately. If $p \nmid k$ then also $p \nmid(k-1-2 \mu)$ and it is straightforward to show that $(-(k-1-2 \mu) \mid 2 \mathbf{1}) \in\left\langle\left(2 \mathbf{1}^{T} \mid 2 J-4 B_{x}-(k+1-2 \mu) I\right)\right\rangle_{p}$ and $\mathbf{1} \in\left\langle 4 B_{x}+(k+1-2 \mu) I\right\rangle_{p}$ and the result follows.

If $p \mid k$ then also $p \mid(1+2 \mu)$. One can show that $\mathbf{1} \in\left\langle B_{x}+\frac{1}{4}(k+1-2 \mu) I\right\rangle_{p}$ and $\mathbf{1} \in\left\langle B_{x}+\frac{1}{4}(k+1-2 \mu) I-\frac{1}{2} J\right\rangle_{p}$ (which finishes the proof) by considering a partition of $B_{x}$ induced by a partition of $\overline{\Gamma(x)}$ consisting of three parts: a vertex, the neighbors of that vertex and the vertices at distance two. For both matrices we add up the rows in each of the 
three parts which gives three vectors for each matrix. When calculating modulo $p$, the all-1 vector is clearly contained in the span of these three vectors in both cases.

Example 4.2 The Johnson graph $J(6,3)$ is the unique distance-regular graph on 20 vertices with intersection array $\{9,4,1 ; 1,4,9\}$. So $J(6,3)$ is a Taylor graph and has spectrum $9^{1}, 3^{5},-1^{9},-3^{5}$. The relevant $p$-ranks are $r k_{2}(A+I)$ and $r k_{3}(A)$. The neighbor graph of any vertex of $J(6,3)$ is the Paley graph $P(9)$ which is self-complementary. So for $J(6,3)$ we have $r k_{2}(A+I)=2+4=6$ and $r k_{3}(A)=9+0+4=13$. According to Haemers and Spence [8] there are nine graphs with the same spectrum as $J(6,3)$ with the following ranks:

\begin{tabular}{rrrrrrr}
\hline & $\mid$ Aut $(\Gamma) \mid$ & $r k_{2}(A+I)$ & $r k_{3}(A)$ & \\
\hline 1 & 1440 & 6 & 13 & 0 & $J(6,3)$ \\
2 & 96 & 8 & - & 13 & 0 & \\
3 & 32 & 8 & - & 13 & 0 & \\
4 & 16 & 10 & - & 13 & 0 & \\
5 & 48 & 10 & - & 13 & 0 & \\
6 & 12 & 10 & - & 13 & 0 & \\
7 & 5 & 9 & 1 & 14 & 0 & \\
8 & 2 & 10 & - & 14 & 0 & \\
9 & 4 & 10 & - & 14 & 0 & \\
\hline
\end{tabular}

\section{References}

1. A.E. Brouwer, "Distance-regular graphs of diameter 3 and strongly regular graphs," Discrete Math. 49 (1984), 101-103.

2. A.E. Brouwer, A.M. Cohen, and A. Neumaier, Distance-Regular Graphs, Ergebnisse der Mathematik 3.18, Springer, Heidelberg, 1989.

3. A.E. Brouwer and C.A. van Eyl, "On the $p$-rank of the adjacency matrices of strongly regular graphs," J. Alg. Combin. 1 (1992), 329-346.

4. A.E. Brouwer and H.A. Wilbrink, "Ovoids and fans in the generalized quadrangle $Q(4,2)$, , Geom. Dedicata 36 (1990), 121-124.

5. E.R. van Dam, "Regular graphs with four eigenvalues," Linear Algebra Appl. 226-228 (1995), $139-162$.

6. C.D. Godsil, "Modular Bose-Mesner Algebras," Preprint, 1999.

7. W.H. Haemers, "Distance-regularity and the spectrum of graphs," Linear Algebra Appl. 236 (1996), 265-278.

8. W.H. Haemers and E. Spence, "Graphs cospectral with distance-regular graphs," Linear and Multilinear Algebra 39 (1995), 91-107.

9. E.S. Lander, Symmetric Designs: An Algebraic Approach, London Math. Soc. Lect. Notes Series 74, Cambridge Univ. Press, Cambridge, 1983.

10. R. Peeters, "Ranks and structure of graphs," Ph.D. Thesis, Tilburg University, 1995.

11. R. Peeters, "Uniqueness of strongly regular graphs having minimal p-rank," Linear Algebra Appl. 226-228 (1995), 9-31.

12. R.M. Wilson, "A diagonal form for the incidence matrices of $t$-subsets vs. $k$-subsets," European J. Combin. 11 (1990), 609-615. 